\title{
Markov chain based on neutrosophic numbers in decision making
}

\author{
Kuppuswami Govindan $^{1}$, Sujatha Ramalingam ${ }^{2, *}$, Nagarajan Deivanayagampillai ${ }^{3}$ \\ Said Broumi ${ }^{4}$, Kavikumar Jacob ${ }^{5}$ \\ ${ }^{1}$ Dept. of Mathematics, Sri Venkateswaraa College of Technology, India. \\ ${ }^{2}$ Dept. of Mathematics, Sri Sivasubramaniya Nadar College of Engineering, India. \\ ${ }^{3}$ Dept. of Mathematics, Hindustan Institute of Technology and Science, India. \\ ${ }^{4}$ Dept. of Mathematics, Faculty of Science Ben MSik, University Hassan II, Morocco. \\ ${ }^{5}$ Dept. of Mathematics and Statistics, Universiti Tun Hussein Onn, Malaysia. \\ *Corresponding author: sujathar@ssn.edu.in
}

\begin{abstract}
Markov chain is a stochastic model for estimating the equilibrium of any system. It is a unique mathematical model in which the future behavior of the system depends only on the present. Often biased possibilities can be used over biased probabilities, for handling uncertain information to define Markov chain using fuzzy environment. Indeterminacy is different from randomness due to its construction type where the items involved in the space are true and false in the same time. In this context as an extension of conventional and fuzzy probabilities neutrosophic probability (NP) was introduced. These neutrosophic probabilities can be captured as neutrosophic numbers. In this paper, Markov chain based on neutrosophic numbers is introduced and a new approach to the ergoticity for the traffic states in the neutrosophic Markov chain based on neutrosophic numbers is verified. The proposed approach is applied to decision-making in the prediction of traffic volume.
\end{abstract}

Keywords: Equilibrium condition; ergotic neutrosophic Markov chain; neutrosophic Markov chain; neutrosophic numbers; traffic.

\section{Introduction}

In recent years, the volume of traffic is one of the major problems faced by developing nations. For solving the congestion problem it is essential to focus on the reasons and take appropriate measures for the regulation of traffic. Any remedial measure should consider the amount of traffic in the future. It is important to analyze the equilibrium state to determine the amount of traffic in the long run. This is done by fitting a suitable mathematical model.

Markov chain is a stochastic model for estimating the long run of any system. A stochastic process with a finite number of states satisfying the memory-less property is called Markov chain (Sheldon M. Ross, 2010) and is widely applied in many real-time problems (Kazemi et al. (2011)). Mainly it is used in the prediction of the future trend of an organization. To analyze the equilib- rium behavior, we consider the system when each input is different and unconditional.

Markov chain (MC) is a unique mathematical model in which the future behavior of the process depends only on the present. This model, consisting of an ensemble of random processes with the possible values from the random variables called the states and their collection called state space. If the system consists of finite-state space then it is discrete time MC with the probabilities between the state transitions modeled using conditional prob-abilities. These probabilities are captured in the form of transition probability matrix (TPM).

The TPM is $n \times n$ if the MC has ' $n$ ' states. The state vector is used to track the position of the states (Fort et al. (2008)). Olaleye et al. (2009) used the Markov approach for the dynamics of vehicular traffic. The Markov chain using Monte 
Carlo for studying the road interconnectivity by Manley (2015). Ning (2013) analyzed the disturbance of traffic flow along a freeway em segment. Rui et al.(2017) discussed the traffic jam by considering their interdependency. He also due to heavy vehicles. A case study on modern establishes a method for Reuse Strategic Decision urban transport sus-tainability assessment by Syed Imran Hussain Shah et al. (2020).

Problems involving traffic flow need to deal with uncertain or insufficient data. Decision making problems in real-time often involve partial inde-terminacy and/or partial determinacy. This is be-cause of lack of data or other factors. Even though fuzzy sets introduced by Zadeh (1965) have the capability to handle uncertain information and ap-plied widely (Koukol et al. (2015); Kaufmann et al. (1985); Hanss, (2015)), fuzzy numbers cannot represent data with both determinate and indeter-minate information. Neutrosophic number $(\mathrm{NN})$ captures determinate and indeterminate in the form of $\mathrm{z}=\mathrm{c}+\mathrm{dI}$, where $\mathrm{c}, \mathrm{d}$ are real numbers and 'I' denotes indeterminacy (Smarandache 1998, $2013,2014)$. NNs have been widely applied in deci-sion making by $\mathrm{Ye}(2016,2017)$ and fault diag-noses (Kong et al. (2015); Ye, (2016)). Pranab et al.(2018) proposed interval trapezoidal neutro-sophic number and specified some arithmetic oper-ations on interval trapezoidal neutrosophic number later considered multiple attribute decision making (MADM) problem with interval trapezoidal neutro-sophic numbers. Irfan Deli (2017) presented a new approach on intervalvalued neutrosophic soft sets denoted by ivn-soft sets are defined and its used to generalize concepts such as soft set, fuzzy soft set, interval-valued fuzzy soft set, intuitionistic fuzzy soft set, intervalvalued intuitionistic fuzzy soft set and neutrosophic soft sets. He also defines a unique notation for expansion and reduction of the neutrosophic classical soft sets are constructed with real-life illustration (Irfan Deli (2018)). A singlevalued trapezoidal neutrosophic (SVTN) numbers with their properties are explained and various aggregation operators are defined by Irfan Deli et al. $(2017,2018)$. To find an alternate way for the decision making problems, a new path was laid down by Irfan Deli (2018) called interval-valued neutrosophic parameterized interval-valued neutrosophic soft sets (ivnpivn-soft sets) and also several other soft sets are generalized for this notion.

Mohammed et al.(2018) developed an integrated framework presented via interval-valued neutrosophic sets to deal with vague, imprecise, and in- consistent information that exists usually in real world and the analytic network process (ANP) is employed to know the weights of selected criteria establishes a method for Reuse Strategic Decision ANP and TOPSIS techniques, enabled by the OSM model with data analytics. This concept helps in statistical data mining, knowledge and heuristic discovery and finally domain transference (Mohammed et al.(2018)). In another study, he used neutrosophic set for decision making to analyze the factors which influence the selection of SCM suppliers (Mohammed et al.(2018)). This method is considered as a proactive approach to improve performance and achieve competitive advantages. A real-time application on the multicriteria group decision-making technique is figured on neutrosophic VIKOR method, for evaluating e-government websites and to represent preferences of decision-makers about criteria significance weights and performance assessments, triangular neutrosophic numbers which are applicable for linguistic variables (Mohammed et al. (2018)). Another real-life problem of an efficient model on neutrosophic analytic hierarchy process is used to solve the performance estimation problem and improve the quality of services by creating a strong competition between cloud providers (Mohammed et al.(2018)). Mohammed et al. (2018) developed another method to evaluate the decision making problem in such a way that each pairwise comparison judgments are symbolized as a trapezoidal neutrosophic number and increasing the number of an alternative in this model.

Often biased possibilities can be used over biased probabilities for handling uncertain information to define MC using a fuzzy environment (Smarandache, (2013)). Markov chains are widely applied in the control system in motor vehicles, regulation of traffic, currency exchange rate, and queuing system. Indeterminacy is different from randomness due to its construction type where the items involved in the space are true and false at the conventional and fuzzy probabilities neutrosophic probability (NP) was introduced. Neutrosophic variable $(\mathrm{NV})$ is subject to change due to randomness and indeterminacy in contrast to the conventional stochastic (random) variable is accounted only for the changes due to randomness. The values of the NV represent the possible outcomes and same time. In this context as an extension of 
indeterminacies which are impartial or partial.

Neutrosophic random process (NRP) performs the change over time of some neutrosophic random values, a collection of neutrosophic random variables(Smarandache, (1998)). A neutrosophic random variable (NRV) is a variable that has vague and ambiguous outcomes (indeterminate). NRV can be either discrete or continuous. The conventional probability and neutrosophic probability will coincide in an experiment in which the chance of getting indeterminacy of a random process is zero. To handle imprecise state, fuzzy state and fuzzy transition probability (FTP) are used. Generally in the applications of MC, data will be collected by assessment or experience and this makes the data incomplete. This kind of problems needs to be handled by fuzzy MC (FMC), where FTP is the base of the FMC, whereas traditional MC is unable to deal with and analyze impreciseness in the decision-making problem. In FMC, TP will be a fuzzy number (Juan et al. (2008); Periyakumar et al. (2016)). The concept of neutrosophic set contributes a new base for handling issues related to indeterminate data which may be numbers or neutrosophic numbers. The clarity of the study can be obtained by neutrosophic probability distributions.

Garcia et al. (2010) made a simulation study on MC under fuzzy environment. Mallak et al. (2011) studied ergodicity of the fuzzy MC using maxmin composition. Sujatha (2012) introduced intuitionistic Markov chain and its path transition and future behavior. Smarandache et al. (2014) introduced the concepts of measurement, integral, and probability under a neutrosophic environment. Smarandache et al. (2013) introduced new concepts such as neutrosophic measure, neutrosophic integral, and the neutrosophic probability in it.

Vajargah et al. (2014) applied Faure and Kronecker sequences to generate the membership values of fuzzy MC and found the number of ergodic MCs. Kanyinda et al. (2015) introduced a method to calculate fuzzy eigenvalues and eigenvectors of a fuzzy MC. Lei et al. (2016) proposed a prediction algorithm for multi aggregation and occasional demand forecasting with fuzzy MC.

Smarandache et al. (2016) applied PCR5 and 2.1 Markov chain

probability under neutrosophic environment to identify the target. Periyakumar et al. (2016) studied about the ergodic behavior of the FMCs. Garcia et al. (2016) proposed quasi MCs under type-2 fuzzy environment. Zhu et al. (2016) provided the sufficient conditions for the ergodicity of fuzzy MCs. Liu et al. (2017) applied MC method in geochemical inverse problems. Awiszus et al. (2018) applied the concept of MC in neural networks. Alhabib et al. (2018) proposed some of the concepts in neutrosophic probability distributions. Petrov et al.(2019) described aggregated MC and applied it in rule-based designing. Broumi et al. (2019) stud-ied the shortest path problem under crisp, fuzzy, intuitionistic, and neutrosophic environments as an overview. Broumi et al. (2019) solved a shortest path problem using interval triangular and trapezoidal neutrosophic environments. Broumi et al. (2019) extended Bellman algorithm under interval neutrosophic environment.

Nagarajan et al. (2019) proposed Dombi interval valued neutrosophic graph and it is operational laws. Nagarajan et al. (2019) studied traffic control management under interval type-2 fuzzy and interval neutrosophic environments. From this literature study, it is evident that Markov chain concept has not been studied using neutrosophic numbers to capture neutrosophic probabilities and thus serves as a motivation for this work.

The required basic concepts are briefed in Section 2. The proposed approach of Markov chain based on neutrosophic numbers is presented in Section 3. In Section 4 the foundation for the classification of traffic states using neutrosophic Markov chain based on neutrosophic numbers is illustrated. Decision making in traffic prediction using the proposed approach and the ergotic verifications for the traffic states based on neutrosophic numbers is discussed in Section 5. Comparative analysis between the proposed method and the existing method and the comparison of the equilibrium condition is given in Section 6 and finally concluded in Section 7.

\section{Basic concepts}

In this section, some of the basic concepts required for the present study have been given.

A Markov chain is a sequence of random variables $X=X_{0}, X_{1}, X_{2}, \ldots$ with the following properties. For, $n \in 0,1,2, \ldots, X_{n}$ is defined on the sample space $\mho$ and takes values from the finite set $S$. Thus $X_{n}: \mho \rightarrow S$. Also for $n \in 0,1,2, \ldots$ and $\left\{i, j, i_{n-1}, i_{n-2}, \ldots, i_{0}\right\} \subseteq S$ 


$$
\begin{aligned}
& P\left\{X_{n+1}=j / X_{n}=i, X_{n-1}=i\right. \\
& \left.\quad-1, X_{n-2}=i-2, \ldots, X_{o}=i_{0}\right\} \\
& \quad=P\left\{X_{n+1}=j / X_{n}=i\right\}
\end{aligned}
$$

and the transition probabilities are independent of $P\left\{X_{n+1}=j / X_{n}=i\right\}=p_{i j}$ are independent of $n$.

\subsection{Neutrosophic set}

Consider the space $X$ consists of universal elements characterized by $X$. The NS is a phenomenon which has the structure

$$
N=\left\{\left(T_{N}(x), I_{N}(x), F_{N}(x)\right) / x \in X\right\}
$$

where the three grades of memberships are from $X$ to $]^{-} 0,1^{+}$of the element $x \in X$ to the set $X$ with the criterion:

${ }^{-} 0 \leq T_{N}(x)+I_{N}(x)+F_{N}(x) \leq 3^{+}$. The functions $T_{N}(x), I_{N}(x)$, and $F_{N}(x)$ are the truth, indeterminate and falsity grades lies in real standard/non-standard subsets of $]^{-} 0,1^{+}[$.

\subsection{Neutrosophic Markov chain}

A neutrosophic stochastic process $\{X(n): n \in \mathbb{N}\}$ is said to be a neutrosophic Markov chain if it satisfies the Markov property

$$
\begin{aligned}
& \beta\left(X_{n+1}=j / X_{n-1}=i, X_{n}=k, \ldots, X_{0}=m\right) \\
& =\beta\left(X_{n+1}=j / X_{n-1}=i\right)
\end{aligned}
$$

where $i, j, k$ establish the state space $S$ of the process. Here $P_{i j} \widetilde{P_{i j}}=\beta\left(X_{n+1}=j / X_{n}=i\right)$ are called the intuitionistic probabilities of moving from state $i$ to state $j$ in one step. Hence $P_{i j} \widetilde{P_{i j}}=$ $\left(T_{P_{i j} \widetilde{P_{i j}}}, I_{P_{i j} \widetilde{P_{i j}}}, F_{P_{i j} \widetilde{P_{i j}}},\right)$, where $T_{P_{i j} \widetilde{P_{i j}}}$ is the truth membership of the transition from state $i$ to state $j$ and $I_{P_{i j} P^{i j}}^{\sim}$ is the indeterminate membership of the transition from state $i$ to state $j$ and $F_{P_{i j}} \underset{P}{\sim}$ is the falsity membership of the transition from state $i$ to state $j$. The matrix $P=\left(P_{i j} \widetilde{P_{i j}}\right)$ is called the neutrosophic transition probability matrix.

\subsection{Operations on neutrosophic numbers}

Let $Z$ denote the set of all real neutrosophic numbers. For any two NNs $z_{1}=a_{1}+u_{1} I$ and lows:

$$
\begin{aligned}
z_{1}+z_{2}= & a_{1}+a_{2}+\left(u_{1}+u_{2}\right) I \\
= & {\left[a_{1}+a_{2}+u_{1} \text { inf } I+u_{2} \text { inf } I, a_{1}+a_{2}\right.} \\
& \left.+u_{1} \text { supI }+u_{2} \text { supI }\right]
\end{aligned}
$$

$$
\begin{aligned}
z_{1}-z_{2}= & a_{1}-a_{2}+\left(u_{1}-u_{2}\right) I \\
= & {\left[a_{1}-a_{2}+u_{1} \text { inf } I-u_{2} \text { inf } I, a_{1}-a_{2}\right.} \\
& \left.\quad+u_{1} \text { supI }-u_{2} \text { supI }\right]
\end{aligned}
$$

$z_{1} \times z_{2}=a_{1} a_{2}+\left(a_{1} u_{2}+a_{2} u_{1}\right) I+u_{1} u_{2} I^{2}$

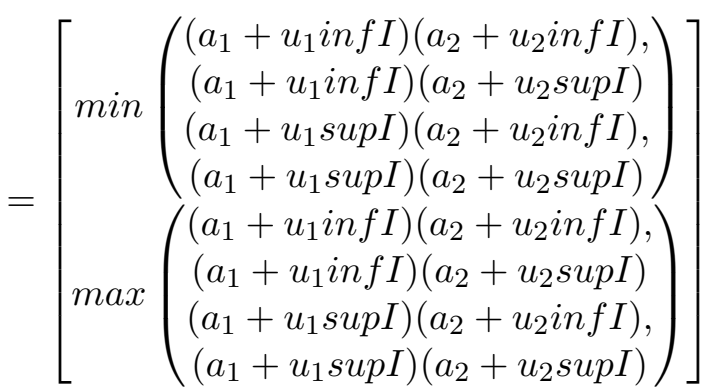

$\frac{z_{1}}{z_{2}}=\frac{\left(a_{1}+u_{1} I\right)}{\left(a_{2}+u_{2} I\right)}=\frac{\left[a_{1}+u_{1} \text { inf } I, a_{1}+u_{1} \text { supI }\right]}{\left[a_{2}+u_{2} \text { infI, } a_{2}+u_{2} \text { supI }\right]}$

$\left[\begin{array}{l}\min \left(\frac{a_{1}+u_{1} \text { infI I }}{a_{2}+u_{2} \text { supI }}, \frac{a_{1}+u_{1} \text { inf } I}{a_{2}+u_{2} \text { supI }}, \frac{a_{1}+u_{1} \text { supI }}{a_{2}+u_{2} \text { supI }}, \frac{a_{1}+u_{1} \text { supI }}{a_{2}+u_{2} \text { supI }}\right) \\ \max \left(\frac{a_{1}+u_{1} \text { infI }}{a_{2}+u_{2} \text { supI }}, \frac{a_{1}+u_{1} \text { infI }}{a_{2}+u_{2} \text { supI }}, \frac{a_{1}+u_{1} \text { supI }}{a_{2}+u_{2} \text { supI }}, \frac{a_{1}+u_{1} \text { supI }}{a_{2}+u_{2} \text { supI }}\right)\end{array}\right]$

In the next section, the proposed approach is presented.

\section{Markov chain based on Neutrosophic num- bers}

A mathematical model with a set of states and the conditional probabilities of transition between them satisfying memory-less property is called a Markov chain. If these probabilities are neutrosophic probabilities, then we have a neutrosophic Markov chain. Neutrosophic probability $(N P)$ of an event $E$ is defined as $N P(E)=($ chance that the event may occur, indeterminate chance between the event occur, chance that the event may not occur)(Smarandache. (2013)).

Mathematically, a sequence of neutrosophic random variables $X_{n}$ is said to be a neutrosophic Markov chain if it satisfies the memoryless property given by $N P\left(X_{n+1}=j / X_{n}=\right.$ $\left.i, X_{n-1}, \ldots, X_{0}\right)=N P\left(X_{n+1}=j / X_{n}=i\right)$. 
This means the future state depends only on the present and not the past. The conditional neutrosophic probability $N P\left(X_{n+1}=j / X_{n}=i\right)=$ $(N P)_{i j}$ is called the transition neutrosophic probability from state $i$ to state $j$. If the system has $s$ states, then these probabilities can be represented by a $s \times s$ square matrix $N P$ called the neutrosophic transition matrix (NTM).

The transition neutrosophic probability from state $\mathrm{i}$ to state $\mathrm{j}$ denoted by $(N P)_{i j}$ is taken in the form of neutrosophic number $(N P)_{i j}=a_{i j}+b_{i j} I$ where $a_{i j} \in[0,1], b_{i j} \in[0,1], I \in[0,1]$. Here we are representing neutrosophic probability using neutrosophic numbers, so it will be appropriate to choose the components of the neutrosophic number to lie in the interval $[0,1]$. Since we are dealing with neutrosophic probabilities the indeterminacy value should be very small. The calculations of powers of the neutrosophic transition matrix use the arithmetic operations on neutrosophic numbers given below.

\subsection{Neutrosophic probability after k-steps}

Let $N P(0)=\left(N P_{1}(0) N P_{2}(0) \ldots N P_{s}(0)\right)$ denote the initial neutrosphic vector, where $N P_{i}(0)$ denote the neutrosophic probability of being in state $i$ initially at time step zero. Then the neutrosophic probability of being in state $j$ after $k$ time steps is $N P(k)=\left(N P_{1}(k) N P_{2}(k) \ldots N P_{s}(k)\right)$. This can be calculated using matrix multiplication of neutrosophic numbers as

$$
N P_{j}(k)=\sum_{i=1}^{s} N P_{i}(k-1) N P_{i j}
$$

Hence, $N P(k)=N P(k-1) N P$, where $N P$ is the neutrosophic transition matrix. Also note that, $N P(k)=N P(k-1) N P=N P(k-2)(N P)^{2}$.

\subsection{Equilibrium study of neutrosophic Markov chain}

It is significant to analyze the equilibrium study of any system.

$\lim _{k \rightarrow \infty} N P(k)=\lim _{k \rightarrow \infty} N P(k-1) N P$. This becomes, $E N P=(E N P) N P$, where neutrosophic row vector $E N P$ denotes equilibrium situation of neutrosophic Markov chain.

\section{Classification of States}

Irreducible neutrosophic Markov Chain:A neutrosophic Markov chain based on neutrosophic numbers are said to be irreducible if every state can be reached from every other state in a finite number of steps. In other words, $N P_{i j}^{(n)}>0$ for some $n$ and for all $i$ and $j$. The transition probability matrix of an irreducible neutrosophic Markov chain is an irreducible matrix. Otherwise, the neutrosophic Markov chain is said to be non-irreducible. Here the concept of irreducible in neutrosophic Markov chain is well-defined since all the entries of $N P_{i j}^{(n)}$ is of the form $a+I b$ where $a, b \in[0,1]$ and $I \in[0,1]$.

Example: Consider the current traffic situation as a set of states, (i.e.) $S=$ $\{$ Low, Medium, High, VeryHigh $\}$. The neutrosophic transition matrix for the state- $S$ is given by $\mathrm{NP}=$

$$
\left[\begin{array}{ccc}
0.01+12.5 I & 0.85+0.5 I & \\
& I & 0+0 I \\
0.01+16 I & 0.01+I & \\
& 0.56+I & 0.23+I \\
0+0 I & 0.01+21.49 I & \\
& I & 0.76+0.5 I \\
0+0 I & 0.01+3.5 I & \\
& 0.95+0.5 I & I
\end{array}\right]
$$

where, $I \in[0,0.01]$, and the corresponding state transition diagram is given in Figure-1. In the $\mathrm{NTM}$, the value $0+0 I$ means, there is no possibility of state transition from the state 'High' to the state 'Low' directly. Here all the states are clearly irreducible and it will perfectly match the current traffic scenrio in the sense, if the traffic is very high it will not go to the state 'Low' directly, either it will go the state 'High' or it will go to the state 'Medium' and then it will come to the state 'Low' in a finite no of steps and it is clearly illustrated in Figure-1.

Periodicity: For a recurrent state i, $N P_{i i}^{(n)}>$ 0 for all $n$. We define the period as $\mu_{i}=$ $G C D\left\{n: N P_{i i}^{(n)}>0\right\}$, here $G C D$ denotes the greatest common divisor. In $S$, the state $L o w-L$ is said to be periodic with period $\mu_{i}$ if $\mu_{i}>1$ and aperiodic if $\mu_{i}=1$ and similarly for all other states. All the states in the Figure- 4 are aperiodic.

Non-null persistent: If the states of the neutrosophic Markov chain are finite and irreducible then all its states are non-null persistent. All the states in the Figure-1 are non-null persistent.

Ergodic neutrosophic Markov chain: A neutrosophic Markov chain $\{X(n): n \in \mathbb{N}\}$ based on neutrosophic numbers is said to be ergodic if it is 


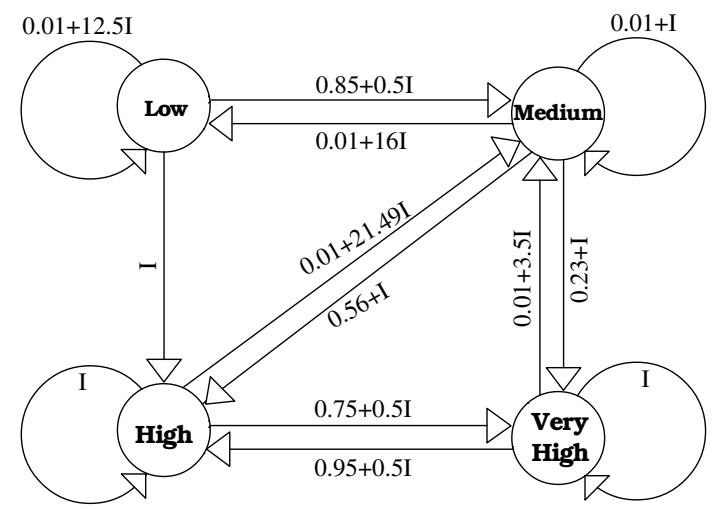

Fig. 1. State transition diagram for the irreducibility of the traffic states

both non-null persistent and aperiodic. The purpose of verifying ergodicity in the neutrosophic Markov chain is to verify the stability of the traffic states and also it guarantees the corresponding neutrosophic Markov chain based on neutrosophic numbers become independent of the initial state and the $n^{t h}$ step as $n \rightarrow \infty$. This implies that $N P^{(n)}$ converges to a neutrosophic matrix with identical rows as $n \rightarrow \infty$. We recollect the following theorems without proof based on the relation between the ergoticity and equilibrium state for the classical Markov and finally we discuss how the theorems perfectly matching with the proposed approach.

Theorem-1: (Sheldon M.Ross 2010) For any irreducible, aperiodic Markov chain, the limiting state probabilities $v_{j}=\lim _{n \rightarrow \infty} p_{j}(n)=$ $\lim _{n \rightarrow \infty} p_{i j}(n)$ exist and are independent of the initial probability vector $p(0)$.

Theorem-2: (Sheldon M.Ross 2010) For an aperiodic Markov chain, the limits $v_{j}=\lim _{n \rightarrow \infty} p_{j}(n)$ exist.

\section{Decision making in traffic prediction}

In recent years, the volume of traffic is one often of the major problems faced by developing nations. For solving the congestion problem it is essential to focus on the reasons and take appropriate measures for the regulation of traffic. Any remedial measure should consider the amount of traffic in the future. It is important to analyze the equilibrium state to determine the amount of traffic in the long run. This is done by fitting a suitable mathematical model. Problems involving traffic flow need to deal with uncertain or insufficient data.

In this work, we consider Velachery-Vijayanagar junction, the three road junction in Chennai, one of

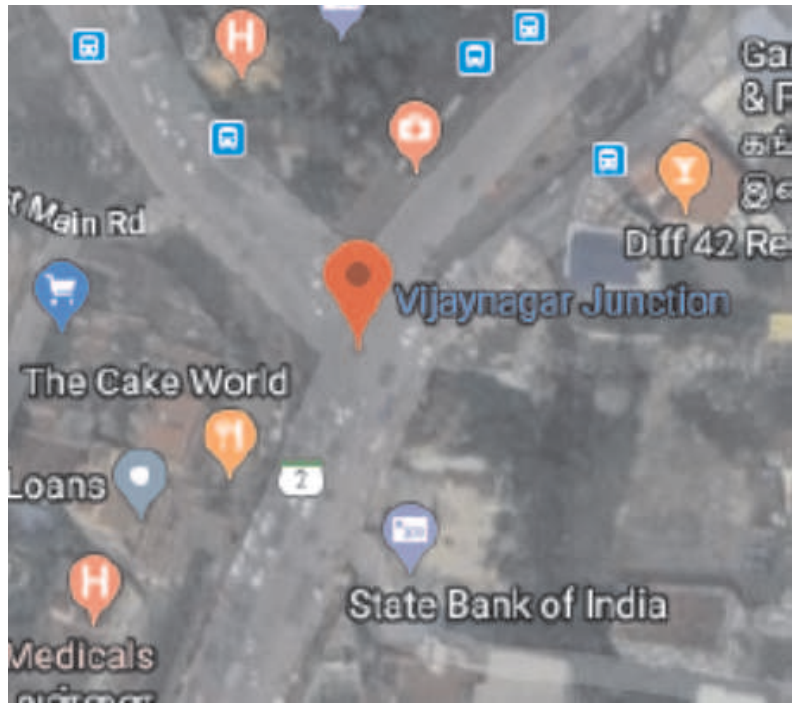

Fig. 2. Google map satellite image for Velachery-Vijayanagar junction

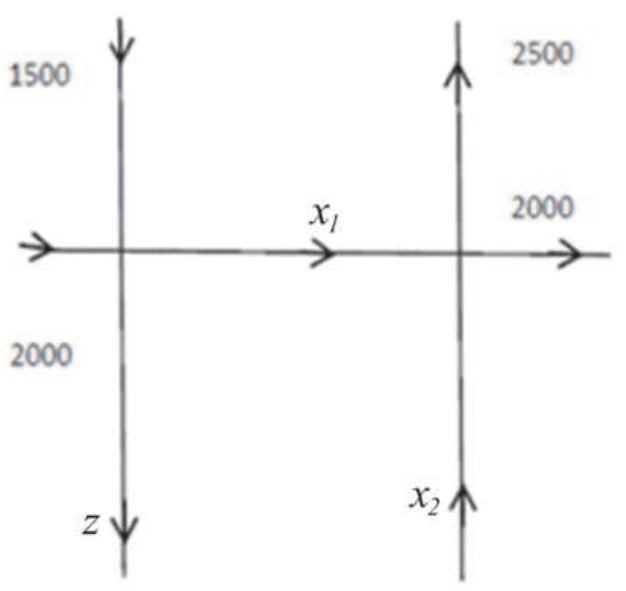

Fig. 3. Traffic flow of three roads

the main cities in India. The Google Maps satellite image is depicted in Figure 1. This junction is one of the busy junctions, with residential complexes, colleges, shopping malls, schools, and bus stands. Thus, it is important to regulate the traffic in this junction to avoid traffic stagnation. To analyze the traffic flow at this junction, the traffic volume was collected for a while in two consecutive years. Based on this data, in this paper, two approaches based on neutrosophic linear equations and Markov chain based on neutrosophic sets are proposed to study traffic flow. Neutrosophic numbers and neutrosophic Markov chain deal effectively with uncertain information. By neutrosophic linear equations, the possible traffic flow volume is calculated. Based on this, to calculate the possible proportion of vehicles in different ranges in the long- term 
neutrosophic Markov chain is used.

5.1 Traffic flow problem analysis using neutrosophic equations

To determine the number of vehicles the concept of neutrosophic linear equations is used. In Figure 2 , we have considered the traffic junction at Chennai. Figure 3, indicates the traffic flow with direction on each one-way road.

Based on the data, the maximum capacity of road 1 is 2000. The flow of traffic is controlled by signals installed at the intersection points. The neutrosophic number $z=500+I$ is a combination of determinate and indeterminate components, where $I$ indicate indeterminacy. The introduction of indeterminacy guarantees no traffic stagnation in the junction. For this, we need to find the range of the number of vehicles flowing in each direction, denoted by $x_{1}, x_{2}$. To ensure no traffic stagnation, all vehicles entering the intersection have to leave the intersection. Due to this condition, we have the following system of neutrosophic linear equations, given by

$$
\begin{gathered}
3500=x_{1}+z ; \\
4500=x_{1}+x_{2} ; \\
z=500+I .
\end{gathered}
$$

Solving the above system, we obtain

$$
\begin{aligned}
& x_{1}=3000-I ; \\
& x_{2}=1500+I .
\end{aligned}
$$

In this junction, the possible traffic flow varies from 600 to 700 (i.e.) $z=[600,700]$, then the range of the traffic flow $x_{1}=[2800,2900]$ and $x_{2}=$ $[1600,1700]$. Based on the data, it was observed that the number of vehicles varies during different time intervals. To reflect this indeterminacy, the possible traffic flow with respect to different ranges is calculated and the results are shown in table 1 .

From table 1, one may note that the traffic flow varies concerning traffic range. To determine the possible proportion of vehicles in different ranges, we use neutrosphic Markov approach. For fitting neutrosophic Markov chain, the traffic volume is counted for some time in two consecutive years and classified. Depending on the total number of vehicles, the states of the neutrosophic Markov chain are defined.
Case-1: The state space is $S=$ $\{$ Low $-L, H i g h-H\}$. The description of the state space is as follows: less than 1000 correspond to state low(L), above 1000 correspond to state high $(\mathrm{H})$. From table 1, the derived neutrosophic Markov chain with neutrosophic transition matrix given by,

$$
N P=\left[\begin{array}{ll}
0.6+0.2 I & 0.3+0.4 I \\
0.4+0.5 I & 0.5+0.3 I
\end{array}\right] \text { Since the in- }
$$

determinacy should be very small, we choose the indeterminacy $I \in[0,0.01]$.

Let $N P=\left[\begin{array}{ll}z_{11} & z_{12} \\ z_{21} & z_{22}\end{array}\right]$ be the matrix. Then, $N P^{2}$ is given by

$$
\begin{array}{r}
{\left[\begin{array}{ll}
z_{11} & z_{12} \\
z_{21} & z_{22}
\end{array}\right]\left[\begin{array}{ll}
z_{11} & z_{12} \\
z_{21} & z_{22}
\end{array}\right]=} \\
{\left[\begin{array}{cc}
z_{11} \times z_{11}+z_{12} \times z_{21} & z_{11} \times z_{12}+z_{12} \times z_{22} \\
z_{21} \times z_{11}+z_{22} \times z_{21} & z_{21} \times z_{12}+z_{22} \times z_{22}
\end{array}\right]}
\end{array}
$$

Then $(1,1)$ element of $N P^{2}$ is given by

$$
N P_{11}^{2}=\left(z_{11} \times z_{11}\right)+\left(z_{12} \times z_{21}\right)
$$

Now,

$$
\begin{aligned}
& z_{11} \times z_{11}= \\
& {\left[\begin{array}{l}
\min ((0.6 \times 0.6),(0.6)(0.6+0.2 \times 0.01), \\
\max ((0.6 \times 0.6),(0.6)(0.6+0.2 \times 0.01), \\
(0.6+0.2 \times 0.01)(0.6), \\
(0.6+0.2 \times 0.01)(0.6), \\
(0.6+0.2 \times 0.01)(0.6+0.2 \times 0.01)) \\
(0.6+0.2 \times 0.01)(0.6+0.2 \times 0.01))
\end{array}\right]} \\
& =\left[\begin{array}{l}
\min (0.36,0.3612,0.3612,0.3624) \\
\max (0.36,0.3612,0.3612,0.3624)
\end{array}\right] \\
& =0.36+0.3624 I
\end{aligned}
$$

$$
\begin{gathered}
z_{12} \times z_{21}= \\
{\left[\begin{array}{l}
\min ((0.3 \times 0.4),(0.3)(0.4+0.5 \times 0.01), \\
\max ((0.3 \times 0.4),(0.3)(0.4+0.5 \times 0.01), \\
(0.3+0.4 \times 0.01)(0.4), \\
(0.3+0.4 \times 0.01)(0.4), \\
\quad(0.3+0.4 \times 0.01)(0.4+0.5 \times 0.01)) \\
\quad(0.3+0.4 \times 0.01)(0.4+0.5 \times 0.01))
\end{array}\right]} \\
=\left[\begin{array}{l}
\min (0.12,0.1215,0.1216,0.123) \\
\max (0.12,0.1215,0.1216,0.123)
\end{array}\right] \\
=0.12+0.123 I
\end{gathered}
$$


Table 1. Traffic flow for different ranges

\begin{tabular}{llll}
\hline$I$ & $z$ & $x_{1}$ & $x_{2}$ \\
\hline 0 & 500 & 3000 & 1500 \\
{$[100,200]$} & {$[600,700]$} & {$[2900,2800]$} & {$[1600,1700]$} \\
{$[200,300]$} & {$[700,800]$} & {$[2800,2700]$} & {$[1700,1800]$} \\
{$[300,400]$} & {$[800,900]$} & {$[2700,2600]$} & {$[1800,1900]$} \\
{$[400,500]$} & {$[900,1000]$} & {$[2600,2500]$} & {$[1900,2000]$} \\
{$[500,600]$} & {$[1000,1100]$} & {$[2500,2400]$} & {$[2000,2100]$} \\
{$[600,700]$} & {$[1100,1200]$} & {$[2400,2300]$} & {$[2100,2200]$} \\
{$[700,800]$} & {$[1200,1300]$} & {$[2300,2200]$} & {$[2200,2300]$} \\
{$[800,900]$} & {$[1300,1400]$} & {$[2200,2100]$} & {$[2300,2400]$} \\
{$[900,1000]$} & {$[1400,1500]$} & {$[2100,2000]$} & {$[2400,2500]$} \\
{$[1000,1100]$} & {$[1500,1600]$} & {$[2000,1900]$} & {$[2500,2600]$} \\
{$[1100,1200]$} & {$[1600,1700]$} & {$[1900,1800]$} & {$[2600,2700]$} \\
{$[1200,1300]$} & {$[1700,1800]$} & {$[1800,1700]$} & {$[2700,2800]$} \\
{$[1300,1400]$} & {$[1800,1900]$} & {$[1700,1600]$} & {$[2800,2900]$} \\
{$[1400,1500]$} & {$[1900,2000]$} & {$[1600,1500]$} & {$[2900,3000]$} \\
{$[1500,1600]$} & {$[2000,2100]$} & {$[1500,1400]$} & {$[3000,3100]$} \\
{$[1600,1700]$} & {$[2100,2200]$} & {$[1400,1300]$} & {$[3100,3200]$} \\
{$[1700,1800]$} & {$[2200,2300]$} & {$[1300,1200]$} & {$[3200,3300]$} \\
{$[1800,1900]$} & {$[2300,2400]$} & {$[1200,1100]$} & {$[3300,3400]$} \\
{$[1900,2000]$} & {$[2400,2500]$} & {$[1100,1000]$} & {$[3400,3500]$} \\
\hline
\end{tabular}

$=((0.36+0.12),(0.36+0.12+0.3624 \times 0.01+$ $0.123 \times 0.01))$

$=(0.48,0.48485524)=0.48+0.48485524 I$

Thus, we get

$$
\begin{aligned}
& N P^{2}=\left[\begin{array}{l}
0.48+0.48485524 I \\
0.44+0.44447525 I
\end{array}\right. \\
& 0.33+0.3333592 I \\
& 0.37+0.37376129 I]
\end{aligned}
$$

$$
N P^{4}=\left[\begin{array}{c}
0.3756+0.379432265 I \\
0.374+0.377815941 I \\
0.2805+0.283361956 I \\
0.1844874+0.186369746 I
\end{array}\right]
$$

$$
N P^{8}=\left[\begin{array}{c}
0.2459824+0.248492132 I \\
0.2459798+0.248489546 I \\
0.1844849+0.18636716 I \\
0.1844874+0.186369746 I
\end{array}\right]
$$

The neutrosophic transition matrix in the equilibrium is

$$
N P^{\infty}=\left[\begin{array}{c}
0.2459824+0.248492132 I \\
0.2459798+0.248489546 I \\
0.1844849+0.18636716 I \\
0.1844874+0.18636974 I
\end{array}\right]
$$

After the seventh iteration the matrix reached an equilibrium state. Equation (22), reveals that LowLow possibility is $0.2459+0.2484 I$ and HighHigh possibility is $0.1844+0.1863 I$, where $I \in$ $[0,0.01]$.

Case-2: In many practical situations the traffic is not always low or high, there is a possibility of transferring from any state(Low, High) to Medium and vice-versa. So, we take this as an important state for the traffic prediction and consider as the second state in $S$. We analyze the equilibrium state and in addition as a new technique the ergoticity is verified in neutrosophic Markov chain based on neutrosophic numbers. The state space is $S=\{$ Low-L, Medium $-M, H i g h-H\}$. Here the description for the state space ' $M$ ' correspond to the range 1000-2000 and the remaining state as mentioned in Case-1. Similarly the neutrosophic transition matrix for this three state is obtained 


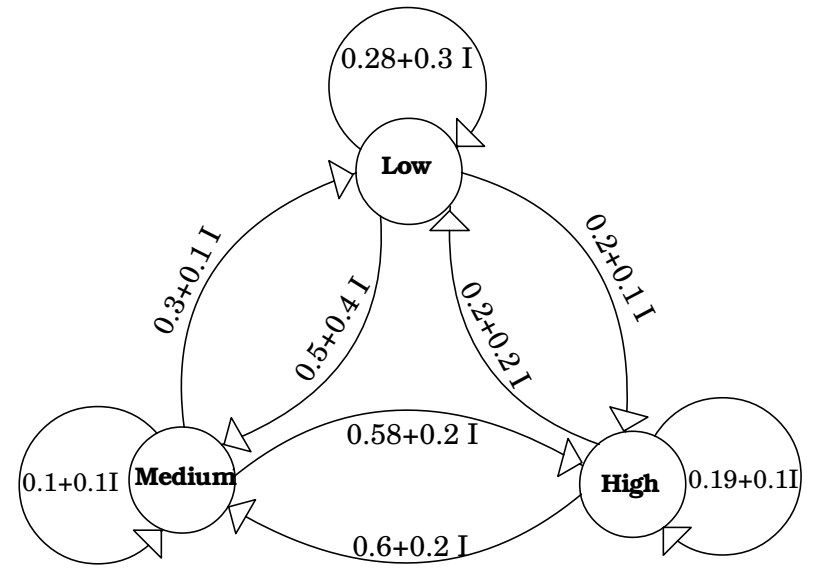

Fig. 4. State transition diagram based on neutrosophic numbers for the traffic states

from table- 1 is given by

$$
N P=\left[\begin{array}{ccc}
0.28+0.3 I & 0.5+0.4 I & 0.2+0.1 I \\
0.3+0.1 I & 0.1+0.1 I & 0.58+0.2 I \\
0.2+0.2 I & 0.6+0.2 I & 0.19+0.1 I
\end{array}\right]
$$

where $I \in[0,0.01]$.

Let $N P=\left[\begin{array}{lll}z_{11} & z_{12} & z_{13} \\ z_{21} & z_{22} & z_{23} \\ z_{31} & z_{32} & z_{33}\end{array}\right]$ be the matrix.

Then, $N P^{2}$ is given by

$$
\left[\begin{array}{l}
z_{11} \times z_{11}+z_{12} \times z_{21}+z_{13} \times z_{31} \\
z_{21} \times z_{11}+z_{22} \times z_{21}+z_{23} \times z_{31} \\
z_{31} \times z_{11}+z_{32} \times z_{21}+z_{33} \times z_{31} \\
z_{11} \times z_{12}+z_{12} \times z_{22}+z_{13} \times z_{32} \\
z_{21} \times z_{12}+z_{22} \times z_{22}+z_{23} \times z_{32} \\
z_{31} \times z_{12}+z_{32} \times z_{22}+z_{33} \times z_{32} \\
z_{11} \times z_{13}+z_{12} \times z_{23}+z_{13} \times z_{33} \\
z_{21} \times z_{13}+z_{22} \times z_{23}+z_{23} \times z_{33} \\
z_{31} \times z_{13}+z_{32} \times z_{23}+z_{33} \times z_{33}
\end{array}\right]
$$

Using (6) and (24) the value of $(N P)^{2}=(N P)(N P)$ is given by

$$
\left[\begin{array}{c}
0.2684+0.2723 I \\
0.23+0.2331 I \\
0.274+0.2769 I \\
0.31+0.3145 I \\
0.508+0.5122 I \\
0.274+0.2775 I \\
0.384+0.388 I \\
0.2282+0.2304 I \\
0.4241+0.4274 I
\end{array}\right]
$$

Similarly proceeding we get the value of $(N P)^{13}=(N P)(N P)^{12}$ is

$$
\left[\begin{array}{l}
0.2117+0.2156 I \\
0.2123+0.2154 I \\
0.2139+0.2172 I \\
0.2847+0.2154 I \\
0.2854+0.2893 I \\
0.2877+0.2920 I
\end{array}\right.
$$

$$
\left.\begin{array}{l}
0.2799+0.2850 I \\
0.2807+0.2847 I \\
0.2828+0.2871 I
\end{array}\right]
$$

$(N P)^{14}=$

$$
\begin{aligned}
& {\left[\begin{array}{l}
0.2082+0.2120 I \\
0.2088+0.2118 I \\
0.2104+0.2136 I
\end{array}\right.} \\
& 0.2800+0.2851 I \\
& 0.2808+0.2849 I \\
& 0.2829+0.2872 I
\end{aligned}
$$

$$
\left.\begin{array}{l}
0.2753+0.2803 I \\
0.2760+0.2800 I \\
0.2781+0.2824 I
\end{array}\right]
$$

$(N P)^{15}=$

$$
\begin{gathered}
{\left[\begin{array}{l}
0.2048+0.2085 I \\
0.2054+0.2083 I \\
0.2069+0.2101 I \\
0.2754+0.2805 I \\
0.2761+0.2801 I \\
0.2782+0.2825 I \\
0.2707+0.2757 I \\
0.2715+0.2754 I \\
0.2735+0.2777 I
\end{array}\right]}
\end{gathered}
$$

$$
(N P)^{16}=
$$

$$
\left[\begin{array}{l}
0.2014+0.2051 I \\
0.2020+0.2049 I \\
0.2035+0.2066 I \\
0.2708+0.2758 I \\
0.2716+0.2755 I \\
0.2736+0.2778 I
\end{array}\right.
$$

$$
\left.\begin{array}{l}
0.2662+0.2711 I \\
0.2670+0.2708 I \\
0.2690+0.2731 I
\end{array}\right]
$$

So $(N P)^{\infty}=$ 


$$
\begin{gathered}
{\left[\begin{array}{l}
0.2014+0.2051 I \\
0.2020+0.2049 I \\
0.2035+0.2066 I \\
0.2708+0.2758 I \\
0.2716+0.2755 I \\
0.2736+0.2778 I \\
0.2662+0.2711 I \\
0.2670+0.2708 I \\
0.2690+0.2731 I
\end{array}\right]}
\end{gathered}
$$

After the sixteenth iteration the matrix reached an equilibrium state. Since we have to give special attention to high traffic volume, here we consider only the possibility of transferring any state to the state High. Equation (30), reveals that the possibility of any state too High is $0.26+0.27 I$, where $I \in[0,0.01]$. These values reflect the amount of traffic in the considered Velechery-Vijayanagar junction. If the same condition prevails, in the future we can control the traffic flow volume for this junction. According to the above results, we can fix the time slot in the traffic signal light system governing this junction.

To understand the concept in a better way, the steady state graph is drawn with the help of the neutrosophic transistion matrix for different values of $I=\{0.0025,0.005,0.0075,0.01\}$. We take the $X-$ axis units from $1-16$, since the matrix reaches steady state after the $16^{\text {th }}$ step and the $Y-a x i s$ is the corresponding probability values. As an example, take $I=0.0025$ and substitute in the 1-step neutrosophic transition matrix (Equation-23), we get one set of nine values (i.e.) $N P_{11}=0.2807, N P_{12}=$ $0.501, N P_{13}=0.2002, N P_{21}=0.3002, N P_{22}=$ $0.1002, N P_{23}=0.5805, N P_{31}=$ $0.2005, N P_{32}=0.6005$ and $N P_{33}=0.1902$. Similarly substituting the value of $I=0.0025$ upto $n=16$, we get '16' sets of '9' values for plotting the graphs. In Figure-5, after $n=16$ all the curves converges to a unique point, (i.e.) the matrix reaches the equilibrium state. Similarly, we proceed with remaining 'I' values.

5.2 Verification of ergoticity for the states of the traffic in neutrosophic Markov chain

Classifications of states: First we verify the irreducibility of the states ' $S$ ' in the traffic. Figure-4 gives the state transition diagram of the $3 \times 3$ neutrosophic matrix based on neutrosophic numbers.

If we consider the first state Low, in the $16^{\text {th }}$ step

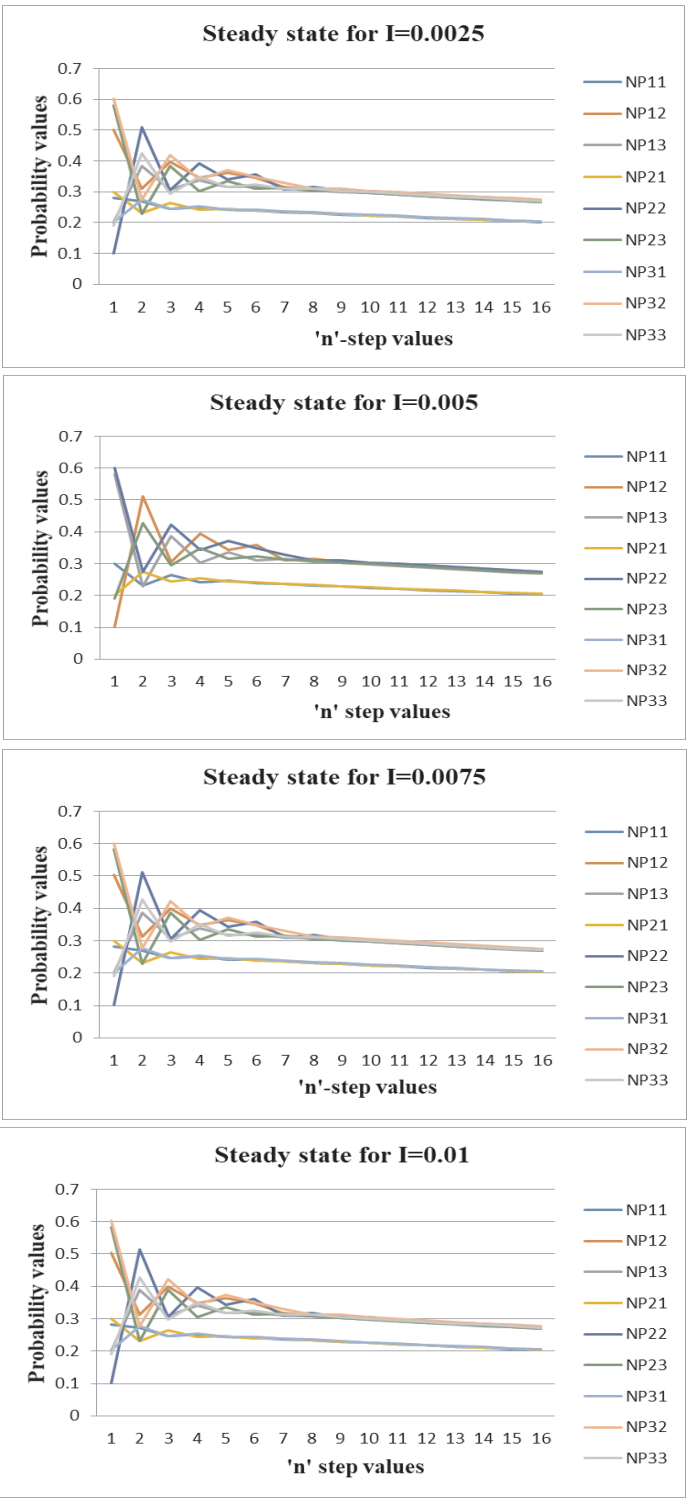

Fig. 5. Steady state for different'I' values

the possibility of being the traffic in the state LowLow is given by $N P_{11}^{(16)}=0.2014+0.2051 I>$ 0 where $I \in[0,0.01]$. In general, the irreducibility of all the traffic states $S$ in the $n^{\text {th }}$ step for $n=$ $1,2,3 \ldots$ is given by

$$
\begin{aligned}
& N P_{11}^{(n)}>0, N P_{12}^{(n)}>0, N P_{13}^{(n)}>0 . \\
& N P_{21}^{(n)}>0, N P_{22}^{(n)}>0, N P_{23}^{(n)}>0 . \\
& N P_{31}^{(n)}>0, N P_{32}^{(n)}>0, N P_{33}^{(n)}>0 .
\end{aligned}
$$

Therefore the neutrosophic Markov chain is irreducible. Next, we find the period of all the traffic states.

For the $1^{\text {st }}$ traffic state 'Low', we have

$$
N P_{11}^{1}>0, N P_{11}^{2}>0, \ldots
$$


Table 2. Equilibrium state of the neutrosophic Markov chain(Method-1)

\begin{tabular}{llll}
\hline$I$ & $N P 1$ & $N P 2$ & $N P 3$ \\
\hline 0.0025 & 0.2019 & 0.2715 & 0.2669 \\
0.005 & 0.2024 & 0.2722 & 0.2676 \\
0.0075 & 0.2029 & 0.2729 & 0.2683 \\
0.01 & 0.2034 & 0.2736 & 0.2690 \\
\hline
\end{tabular}

Table 3. Equilibrium state of the neutrosophic Markov chain(Method-2)

\begin{tabular}{llll}
\hline$I$ & $E N P 1$ & $E N P 2$ & $E N P 3$ \\
\hline 0.0025 & 0.1914 & 0.2883 & 0.2486 \\
0.005 & 0.1918 & 0.2890 & 0.2492 \\
0.0075 & 0.1923 & 0.2897 & 0.2498 \\
0.01 & 0.1928 & 0.2905 & 0.2505 \\
\hline
\end{tabular}

Table 4. Comparison of the Equilibrium states

\begin{tabular}{lllllll}
\hline$I$ & $N P 1$ & $E N P 1$ & $N P 2$ & $E N P 2$ & $N P 3$ & $E N P 3$ \\
\hline 0.0025 & 0.2019 & 0.1914 & 0.2715 & 0.2883 & 0.2669 & 0.2486 \\
0.005 & 0.2024 & 0.1918 & 0.2722 & 0.2890 & 0.2676 & 0.2492 \\
0.0075 & 0.2029 & 0.1923 & 0.2729 & 0.2897 & 0.2683 & 0.2498 \\
0.01 & 0.2034 & 0.1928 & 0.2736 & 0.2905 & 0.2690 & 0.2505 \\
\hline
\end{tabular}

Period of the traffic state

$$
\text { Low }=G C D\{1,2,3, \ldots\}=1 .
$$

For the $2^{\text {nd }}$ traffic state 'Medium', we have

$$
N P_{22}^{1}>0, N P_{22}^{2}>0, \ldots
$$

Period of the traffic state

$$
\text { Medium }=G C D\{1,2,3, \ldots\}=1 .
$$

Similarly for the $3^{\text {rd }}$ traffic state 'High', we have

$$
N P_{33}^{1}>0, N P_{33}^{2}>0, \ldots
$$

Period of the traffic state

$$
H i g h=G C D\{1,2,3, \ldots\}=1 .
$$

All the three traffic states $\{$ Low, Medium, $H i g h\}$ having period 1 . So the three traffic states are aperiodic. The traffic states are finite and irreducible, the neutrosophic Markov chain is nonnull persistent. Here both the conditions, aperiodic and non-null persistent are satisfied and hence the neutrosophic Markov chain is ergodic.
Here both the theorems in the classical Markov is very well matching with the proposed work. As per theorem-1, here we verified that in any irreducible, aperiodic neutrosophic Markov chain based on neutrosophic numbers, the equilibrium state exists for the $3 \times 3$ neutrosophic matrices, theorem-2 is clearly true in neutrosophic Markov, (i.e.) for any aperiodic neutrosophic Markov chain, the equilibrium state exists.

\section{Comparative analysis}

Here the comparative analysis has been done in Table- 5 between the classical Markov and neutrophic Markov based on neutrosophic numbers to understand the newly introduced method in a better way and the major role played by the neutrosophic Markov chain to identify the traffic states in the equilibrium position. Analytically, the equilibrium state of the neutrosophic Markov chain can be obtained as the solution of $(E N P) N P=E N P$, where $E N P$ is a row vector of neutrosophic numbers which constitute the equilibrium state of the neutrosophic Markov chain and NP is the neutrosophic transition matrix. For the neutrosophic transition matrix is given in equation (23), the equilibrium position for different levels of indeterminacy ' $I$ ' is obtained by finding $\lim _{k \rightarrow \infty} N P(k)$, 
Table 5. Comparative analysis between neutrosophic Markov based on neutrosophic numbers with the classical Markov

\begin{tabular}{|c|c|}
\hline S.No & Neutrosophic Markov \\
\hline 1. & $\begin{array}{l}\text { Recently developed method } \\
\text { deals with indeterminacy occurs } \\
\text { in the system. }\end{array}$ \\
\hline 2. & $\begin{array}{l}\text { Very suitable technique for pre- } \\
\text { dicting the traffic, since some of } \\
\text { the concepts for the traffic con- } \\
\text { gestion are indeterminant (Su- } \\
\text { jatha et al. 2019). }\end{array}$ \\
\hline
\end{tabular}

3. Transition between the states are neutrosophic numbers in the NTM. Due to the presence of indeterminacy, NTM is not a stochastic matrix.

4. Neutrosophic transition matrix based on neutrosophic numbers are multiplied by min-max operation.

5. Equilibrium state of the neutrosophic Markov chain using neutrosophic numbers reveals the possibility of the transition of the traffic states accurately to predict the traffic.

6. For the traffic states stability, verification of ergoticity can be reached in minimum number of steps.

\begin{abstract}
Classical Markov
Very traditional method in which

the future depends only on the present. Not suitable for the system where uncertainty and indeterminacy occurs.

Does not have the capability of dealing with indeterminacy of the traffic.
\end{abstract}

Transition between the states have probabilistic values in the TPM. TPM is a stochastic matrix.

Transition probability matrices are multiplied by usual matrix multiplication.

The probability of transition from one traffic state to another in the steady state is less accurate, since some of the traffic concepts are indeterminant.

Irreducibility verification of traffic states itself takes maximum steps compared to the proposed method. 


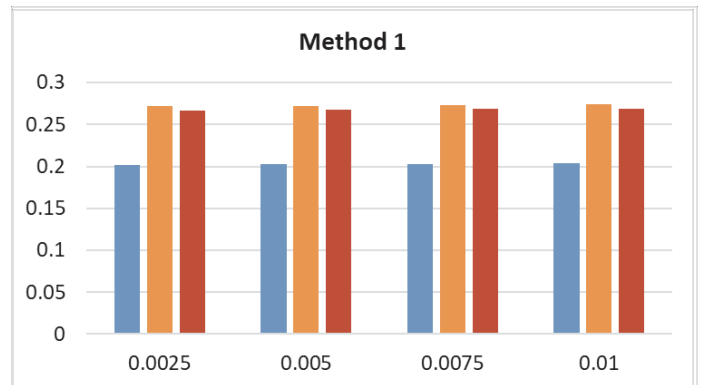

Fig. 6. The equilibrium state for different'I'values (Method-1)

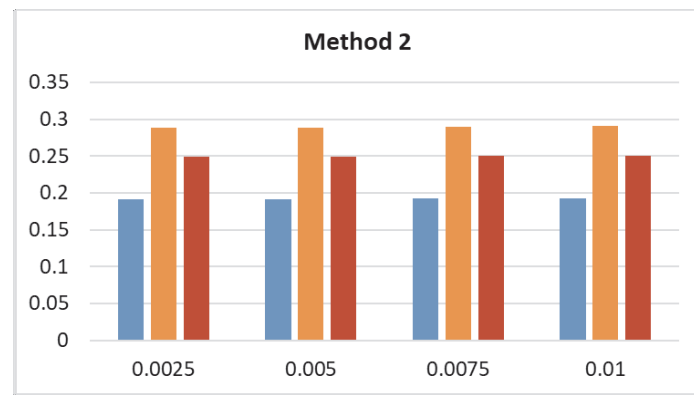

Fig. 7. The equilibrium state for different 'I' values.(Method-2)

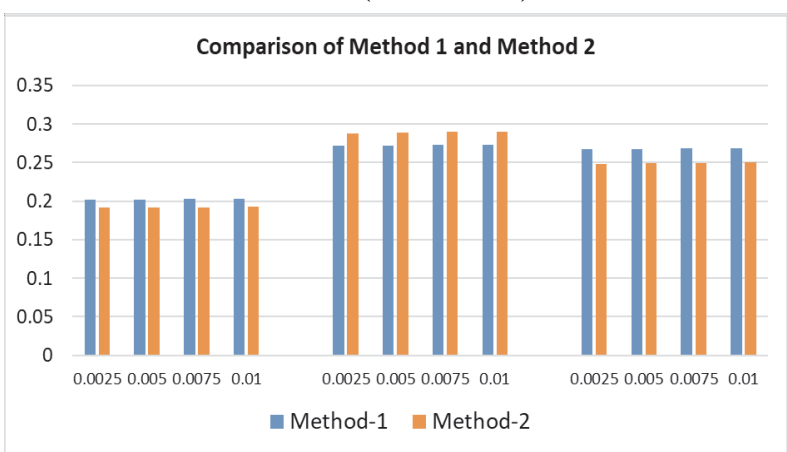

Fig. 8. Comparison of Method-1 and Method-2

(Method-1) and $(E N P) N P=E N P$ (Method-2) is tabulated in table-2 and table- 3 and the corresponding graphs is given in Figure-6 and Figure-7.

Further, the comparison between the equilibrium states for both the methods are presented in table-4. The comparison is depicted in the graph. From the Figure-8, we observe that the equilibrium position by both methods are approximately equal.

\section{Summary and Conclusion}

This paper presents a new neutrosophic Markov model based on neutrosophic numbers. This new mathematical model is applied to predict the traffic. Three traffic states are defined according to the range using the collected real-time traffic volume. The prediction is done by analyzing the equilibrium condition of the system. Through this analysis, the possibility of the changes in the traffic states are also determined. On the other hand, the results of the comparative study for the equilibrium condition in both the methods are approximately equal. All the concepts are illustrated in graphs. Further, to study the stability of the traffic states the ergodic properties are verified for the neutrosophic Markov chain using neutrosophic numbers.

The purpose of the proposed work is as follows: This method provides an effective way in decision-making to manage traffic congestion in an indeterminate environment. This model can be applied to predict and decide traffic flow management in any road junction. We believe this method would be very useful to the transportation department in advance, to identify the traffic condition in any part of the world. Also, the proposed work can be applied in real-life problems like weather prediction, stock marketing etc to make decision. Further, as a research prospect, it can also be enhanced as a hidden Markov model using neutrosophic numbers in decision making.

\section{References}

Alhabib, R., Ranna, M. M., Farah, H. \& Salama, A. A. (2018). Some neutrosophic probability distributions. Neutrosophic Sets and Systems, 22 :30-38.

Awiszus, M. \& Rosenhahn, B.(2018). Markov chain neural networks. IEEE/CVF Conference on Computer Vision and Pattern Recognition Workshops (CVPRW).

Broumi, S., Talea, M., Bakali, A., Smarandache, F., Nagarajan, D., Lathamaheswari, M. \& Parimala, M. (2019). Shortest path problem in fuzzy, intuitionistic fuzzy and neutrosophic environment: an overview. Complex \& Intelligent Systems, 5:371-378.

Broumi, S., Nagarajan, D., Bakali, A., Talea, M., Smarandache, F. \& Lathamaheswari, M. (2019). The shortest path problem in interval valued trapezoidal and triangular neutrosophic environment. Complex \& Intelligent Systems, 5 :391-402.

Broumi, S., Dey, A., Talea, M., Bakali, A.,Smarandache, F., Nagarajan, D., Lathamah eswari, M. \& Kumar, R. (2019). Shortest path 
problem using Bellman algorithm under neutrosophic environment. Complex \& Intelligent Systems, 5 :409-416.

Fort, G., Meyn, S., Moulines, E. \& Priouret, P. (2008). The ODE method for stability of skipfree Markov chains with applications to Markov chain. The Annals of Applied Probability, 18 :664-707.

Garcia, J. C. F. (2010). Interval type-2 fuzzy Markov chains. In Fuzziness and Soft Computing, 301 :1-6.

Garcia, J. C. F., Arcos, L. C. G. \& Rivera, S. K. L. (2016). Quasi type-2 fuzzy Markov chains: An approach. IEEE International Conference on Fuzzy Systems(FUZZ-IEEE).

Hanss, M. (2005). Applied fuzzy arithmetic: an introduction with engineering applications. Springer, Germany.

Irfan Deli. (2017). Interval-valued neutrosophic soft sets and its decision making. International Journal of Machine Learning and Cybernetics, 8 :665-676.

Irfan Deli. (2018). Expansions and reductions on neutrosophic classical soft set. Journal of Natu-ral and Applied Sciences, 22: 478-486.

Irfan Deli \& Yusuf Subas. (2017). Some weighted geometric operators with SVTrN-numbers and their application to multi-criteria decision mak-ing problems. Journal of Intelligent and Fuzzy Systems, 32 :291-301.

Irfan Deli. (2018). Operators on single valued trapezoidal neutrosophic numbers and SVTNgroup decision making. Neutrosophic Sets and Systems, 22 :131-150.

Juan, C., Garcia, F., Kalenatic, D. \& Bello, C. A. L. (2018). A simulation study on fuzzy Markov chains. International Conference on Intelligent Computing, 109-117.

Kanyinda, J. P. M., Matendo, R. M. M., Lukata, B. U. E. \& Ibula, D. N.(2015). Fuzzy eigenvalues and fuzzy eigen vectors of fuzzy Markov chain transition matrix under max-min composition. Journal of Fuzzy Set Valued Analysis, 1 :25-35.
Kaufmann, A. \& Gupta, M. M.(1985). Introduction to fuzzy arithmetic. Van Nostrand Reinhold Company, USA.

Kazemi, A., Modarres, M., Mehregan, M. R., Neshat, N. \& Foroughi, A. A.(2011). Markov chain grey forecasting model: A case study of energy demand of industry sector in Iran. International Conference on Information and Financial Engineering IPEDR, IACSIT Press, Singapore. 12 :13-18.

Kong, L., Wu, Y. \& Ye, J.(2015). Misfire fault diagnosis method of gasoline engines using the cosine similarity measure of neutrosophic numbers. Neutrosophic Sets and Systems, 8 :43-46.

Koukol, M., Zajilkova, L., Marek, L. \& Tulek, P. (2015). Fuzzy logic in traffic engineering: A review on signal control. Mathematical Problems in Engineering, 2015 :1-14.

Lei, M., Li, S. \& Tan, Q. (2016). Intermittent demand forecasting with fuzzy markov chain and multi aggregation prediction algorithm. Journal of Intelligent and Fuzzy Systems, 31 :2911-2918.

Liu, B. \& Liang, Y. (2017). An introduction of Markov chain Monte Carlo method to geochemical inverse problems: Reading melting parameters from REE abundances in abyssal peridotites. Geochimica et Cosmochimica Acta, 203 :216-234.

Mallak, S. F., Marabeh, M. M. A. \& Ziqan, A. (2011). A Particular class of ergodic finite fuzzy Markov chains. Advances in Fuzzy Mathemat-ics, 6(2) :253-268.

Manley, E. (2015). Estimating urban traffic patterns through probabilistic inter-connectivity of road network junctions. Plos One, 10(5) :1-17.

Mohammed, A. B., Manogaran, G., Gamal. A. \& Smarandache, F. (2018). A hybrid approach of neutrosophic sets and DEMATEL method for developing supplier selection criteria. De-sign Automation for Embedded System, 22 : 257278.

Mohammed, A. B., Zhou, Y., Mohammed, M.\& Chang, V. (2018). A group decision making framework based on neutrosophic VIKOR approach for e-government website evaluation. 
Journal of Intelligent and Fuzzy Systems, 34(6) :4213-4224.

Mohammed, A.B., Mohammed, M. \& Chang, V. (2018). NMCDA: A framework for evaluating cloud computing services. Future Generation Computer Systems, 86 :12-29.

Mohammed, A. B., Atef, A. \& Smarandache, F.(2018). A hybrid neutrosophic multiple crite-ria group decision making approach for project selection. Cognitive Systems Research, 1-12.

Nagarajan, D., Lathamaheswari, M., Broumi, S. \& Kavikumar, J.(2019). Dombi interval valued neutrosophic graph and its role in traffic control management. Neutrosophic Sets and Systems, 24 :114-133.

Nagarajan, D., Lathamaheswari, M., Broumi, S. \& Kavikumar, J. (2019). A new perspective on traffic control management using triangular interval type-2 fuzzy sets and interval neutrosophic sets. Operations Research Perspectives, 6:1-13.

Ning, Wu. (2013). A stochastic model for reliability analysis in freeway networks. Procedia-Social and Behavioral Sciences, 96 :2823-2834.

Olaleye, O. T., Sowunmi, F. A., Abiola, O.S., Salako, M. O. \& Eleyoowo, I. O.(2009). A Markov chain approach to the dynamics of vehicular traffic characteristics in Abeokutametropolis. Research Journals of Applied Sci-ences, Engineering and Technology, 1 :160-166.

Petrov, T. (2019). Markov chain aggregation and its application to rule-based modelling. In book: Modeling Biomolecular Site Dynamics, 1945 :297-313.

Periyakumar, J.A., Sreevinotha, V. (2016). On the ergodic behaviour of fuzzy Markov chains. IOSR Journal of Mathematics (IOSR-JM), 12(5) :28-34.

Pranab, B., Surapati, P., Abiola, O. S. \& Bibhas, C. G.(2018). Distance measure based MADM strategy with interval trapezoidal neutrosophic numbers. Neutrosophic Sets and Systems, 19 :40-46.
Rui, J., Cheng, J. J., Zhang, H. M., Huang, Y. X., Tian, J. F., Hu, M. B., Wang, H.\& Jia, B. (2017). Experimental and empirical investi-gations of traffic flow instability. Transportation Research Procedia, 23 :157-173.

Sheldon, M. Ross. (2010). Introduction to probability models, 10th edition. Academic Press an Imprint of Elsevier.

Smarandache, F. (1998). Neutrosophy:Neutrosophic Probability, Set and Logic. American Research Press, Rehoboth, USA.
Smarandache,
F.(2013).
Introduction Neutrosophic Measure, Neutrosophic
Integral and Neutrosophic Sitech \& Educational, Craiova, Columbus. (http://fs.unm.edu/NeutrosophicMeasureIntegral Probability.pdf).

Smarandache, F. (2014). Introduction to Neutrosophic Statistics. Sitech: Craiova, Romania, Education Publishing, USA. (http://fs.unm.edu/NeutrosophicStatistics.pdf)

Smarandache, F., Abbas, N., Chibani, Y., Hadjadji, B. \& Omar, Z. A.(2016). PCR5 and neutrosophic probability in target identification. Progress in Nonlinear Dynamics and Chaos, 4(2) :45-50.

Sujatha, R. (2012). An introduction to intuitionistic Markov chain. International Mathematical Forum, 7(50) : 2449-2456.

Sujatha, R., Kuppuswami, G., Vasantha Kandasamy. W. B. \& Said Broumi. (2019). An approach for study of traffic congestion problem using fuzzy cognitive maps and neutrosophic cognitive maps-the case of Indian traffic. Neutrosophic Sets and Systems, 30 :273-283.

Syed Imran Hussain Shah., Rab Nawaz., Sajjad Ahmad., Muhammad Arshad., Rabiya Nasir., Muhammad Yaseen., Sabiha-Javied, \& Muhammad Atif Irshad. (2020). Sustain-bility assessment of modern urban transport and its role in the reduction of greenhouse gas emissions: A case study of Metro Bus System(MBS), Lahore. Kuwait Journal of Science, 47(2) :67-81. 
Vajargah, B. F. \& Gharehdaghi, M. (2014). Ergodicity of fuzzy Markov chains based on simulation using sequences. Journal of Mathematics and Computer Science, 11 :159-165.

Ye, J. (2016). Multiple-attribute group decisionmaking method based on linguistic neutrosophic numbers. Journal of Intelligent Systems, 25 :377-386.

Ye, J. (2016). Fault diagnoses of steam turbine using the exponential similarity measure of neutrosopic numbers. Journal of Intelligent and Fuzzy Systems, 30(4) :1927-1934.

Ye, J. (2017). Bidirectional projection method for multiple attributed group decision making with neutrosophic numbers. Neural Computing and Applications, 28(5) :1021-1029.

Zadeh, L. A. (1965). Fuzzy sets. Information and Control, 8 :338-353.

Zhu, D. M., Ching, W. K. \& Guu, S. M. (2016). Sufficient conditions for the ergodicity of fuzzy Markov chains. Fuzzy Sets and Systems, 30:82-93.

$\begin{array}{lr}\text { Submitted: } & 30 / 05 / 2020 \\ \text { Revised: } & 01 / 12 / 2020 \\ \text { Accepted: } & 15 / 12 / 2020 \\ \text { DOI: } & 10.48129 / \text { kjs.v48i4.9849 }\end{array}$

